\title{
Sensibilidade moral dos profissionais da atenção primária nas situações de vulnerabilidades relacionadas ao comportamento suicida
}

\author{
Moral sensitivity of primary care professionals in situations of vulnerabilities related to suicidal \\ behavior \\ Sensibilidad moral de los profesionales de atención primaria en situaciones de vulnerabilidades \\ relacionadas con el comportamiento suicida
}

Recebido: 25/03/2021 | Revisado: 03/04/2021 | Aceito: 08/04/2021 | Publicado: 17/04/2021

Micheli Leal Ferreira

ORCID: https://orcid.org/0000-0002-3064-1875

Faculdade SENAC Caçador, Brasil

E-mail: micheli_leal@yahoo.com.br

Mara Ambrosina de Oliveira Vargas

ORCID: https://orcid.org/0000-0003-4721-4260

Universidade Federal de Santa Catarina, Brasil

E-mail: ambrosina.mara@ufsc.br

Jeferson Rodrigues

ORCID: https://orcid.org/0000-0002-8612-9088

Universidade Federal de Santa Catarina, Brasil

E-mail: jeferson.rodrigues@ufsc.br

Francielly Zilli

ORCID: https://orcid.org/0000-0001-9697-2709

Universidade Federal de Santa Catarina, Brasil E-mail: franciellyzilli.to@gmail.com

Daiane Trentin

ORCID: https://orcid.org/0000-0003-3074-3617

Prefeitura de Passo Fundo, Brasil

E-mail: daitrentin@yahoo.com.br

Caroline Porcelis Vargas

ORCID: https://orcid.org/0000-0002-9904-0816

Universidade Federal de Santa Catarina, Brasil

E-mail:k2vargas@gmail.com

\begin{abstract}
Resumo
Objetivo: Analisar atuação dos profissionais da atenção primária à saúde sobre o comportamento suicida na perspectiva conceitual da vulnerabilidade e sensibilidade moral em um município catarinense. Método: Estudo qualitativo mediante entrevista semiestruturada com 19 profissionais da Atenção Primária em Saúde. Os dados foram analisados com base na análise de conteúdo. Resultados: A partir da percepção dos profissionais, foi possível identificar uma visão ampliada quanto à multicausalidade/manifestações do comportamento suicida, ainda, foi possível pontuar que, a abordagem profissional somada à resposta das instituições e coparticipação da família/sociedade podem gerar, perpetuar e transformar vulnerabilidades, e por fim, evidenciou-se insuficiência de ações em saúde, representando oposição ao objetivo de ultrapassar vulnerabilidades. Conclusão: Na sensibilidade moral, perceber o problema deve estar atrelado ao repensar a prática. Inovação das ações e busca por recursos que perpassem barreiras vão em direção à defesa dos direitos da pessoa em sofrimento, rompimento das vulnerabilidades e amenização do sofrimento profissional. O diferencial da problemática do comportamento suicida está na compreensão a partir da perspectiva da vulnerabilidade e sensibilidade moral, que permitiu apontar a necessidade contínua de aprimoramento de habilidades que impactam nas estratégias de intervenção, assim como, direcionar o olhar para o trabalhador o qual passa a lidar com angústias desencadeadas dos problemas éticos.
\end{abstract}

Palavras-chave: Suicídio; Atenção primária à saúde; Desenvolvimento moral; Ética profissional; Populações vulneráveis.

\begin{abstract}
Objective: To analyze the performance of primary health care professionals on suicidal behavior from the conceptual perspective of vulnerability and moral sensitivity in a municipality of Santa Catarina. Method: Qualitative study through semi-structured interviews with 19 primary health care professionals. The data were analyzed based on content analysis. Results: From the perception of professionals, it was possible to identify an expanded view regarding the multicausality/manifestations of suicidal behavior, it was also possible to point out that the professional approach
\end{abstract}


added to the response of institutions and co-participation of family/society can generate, perpetuate and transform vulnerabilities, and finally, there was insufficient health actions, representing opposition to the objective of overcoming vulnerabilities. Conclusion: In moral sensitivity, perceiving the problem must be tied to rethinking practice. Innovation of actions and search for resources that permeate barriers go towards the defense of the rights of the person in suffering, disruption of vulnerabilities and alleviation of professional suffering. The differential of the problem of suicidal behavior lies in understanding from the perspective of vulnerability and moral sensitivity, which allowed us to point out the continuous need to improve skills that impact intervention strategies, as well as to direct the gaze to the worker who begins to deal with anguish triggered from ethical problems.

Keywords: Suicide; Primary health care; Moral development; Ethics professional; Vulnerable populations.

\section{Resumen}

Objetivo: Analizar el desempeño de los profesionales de la atención primaria de salud sobre el comportamiento suicida desde la perspectiva conceptual de vulnerabilidad y sensibilidad moral en un municipio de Santa Catarina. Método: Estudio cualitativo a través de entrevistas semiestructuradas con 19 profesionales de la atención primaria de salud. Los datos se analizaron en función del análisis de contenido. Resultados: A partir de la percepción de los profesionales, fue posible identificar una visión ampliada con respecto a la multicausalidad/manifestaciones del comportamiento suicida, también fue posible señalar que el enfoque profesional añadido a la respuesta de las instituciones y la co-participación de la familia / sociedad puede generar, perpetuar y transformar vulnerabilidades, y finalmente, no hubo suficientes acciones de salud, representando la oposición al objetivo de superar vulnerabilidades. Conclusión: En la sensibilidad moral, percibir el problema debe estar ligado a repensar la práctica. La innovación de las acciones y la búsqueda de recursos que permean barreras se destinan a la defensa de los derechos de la persona en el sufrimiento, la alteración de las vulnerabilidades y el alivio del sufrimiento profesional. El diferencial del problema del comportamiento suicida radica en la comprensión desde la perspectiva de la vulnerabilidad y la sensibilidad moral, lo que nos permitió señalar la necesidad continua de mejorar las habilidades que impactan las estrategias de intervención, así como dirigir la mirada hacia el trabajador que comienza a lidiar con la angustia desencadenada por problemas éticos.

Palabras clave: Suicidio; Atención primaria de salud; Desarrollo moral; Ética profesional; Poblaciones vulnerables.

\section{Introdução}

A cada 40 segundos uma pessoa tira a própria vida, e mais de $50 \%$ das mortes violentas no mundo tem como causa o suicídio (Lucas, Costa, \& Souza, 2017), sendo que, entre os anos de 1990 e 2016 as mortes por suicídio aumentaram globalmente de 0,4\% para 15,6\% (Naghavi, 2019). Globalmente, é a segunda principal causa de morte de pessoas entre 15 e 29 anos, tendo o Brasil como oitavo país com maior número de suicídio (Silva, Prates, Cardoso, \& Rosas, 2018; Oliveira, \& Benedetti, 2018). Santa Catarina (SC) faz parte da região Sul, apesar de reunir $14 \%$ da população, concentrou $23 \%$ dos suicídios no Brasil, entre 2012 e 2017 (Diretoria de Vigilância Epidemiologica [DIVE], 2019).

As representações sociais do suicídio estão articuladas com atos para tirar a própria vida, sempre acompanhadas de ideias periféricas relacionadas com motivação, intencionalidade e letalidade (Ribeiro, Castro, \& Scatena, 2018). Constitui fenômeno complexo e multicausal, sem explicação universal por ser produto de distintas interações entre fatores psicológicos, sociais, biológicos, filosóficos e antropológicos individuais com representações singulares (Ribeiro, Castro, \& Scatena, 2018; Lima, \& Gondim, 2019; Ferracioli et al., 2019).

O suicídio é problema de saúde pública, de difícil manejo com impacto na esfera interpessoal, familiar, social e econômica (Lima, \& Gondim, 2019; Ferracioli et al., 2019; Prata et al., 2017). O comportamento suicida (CS) pode ser descrito por um espectro de manifestações, compreende pensamentos, ameaças, gestos, planos, tentativas e o suicídio consumado (Sartorino et al., 2018).

Situações de vulnerabilidade sugerem o risco de suicídio, citamos: traumas, abusos físicos/psicológicos, dor crônica, transtornos mentais, história familiar de suicídio, tentativa prévia e estigmas/tabus. Consideramos a vulnerabilidade condição susceptível a qualquer pessoa, entretanto a pessoa com CS vivência vulnerabilidade acrescida devido às características biológicas, aspectos socioeconômicos/culturais que exige prudência e vigilância peculiar (Machado, \& Albuquerque, 2019).

Nesse contexto, a sensibilidade moral (SM) compreende a habilidade de perceber e intervir eticamente frente às situações de vulnerabilidades e do CS, com suas distintas formas e manifestações, sendo exercida por uma constante 
autocrítica/autoavaliação da práxis profissional (Machado, \& Albuquerque, 2019). É elemento singular, adquirido com experiências pessoais de "sentir o significado moral" em cada situação, não se prende ao senso comum, mas motiva e leva o profissional a rever suas práticas e articular à teoria constantemente, buscar soluções adequadas na gestão de problemas nos distintos espaços de cuidado, refere-se à atuação pessoal no relacionamento interpessoal (Nora, Schaefer, Álvarez, \& Ramos, 2016).

Pressupõe-se que o adequado cuidado ao CS necessita de conhecimento técnico-científico, mas também, desenvolvimento da SM do profissional. Aplicamos esta hipótese na Atenção Primária em Saúde (APS), por ocupar posição privilegiada, em comparação aos demais serviços da Rede de Atenção Saúde (RAS). Isto se deve à inserção territorial e ao acesso facilitado às pessoas/comunidade, valor estratégico no cuidado ao CS (Prata et al., 2017). Assim, objetivamos analisar a atuação dos profissionais da APS sobre o comportamento suicida na perspectiva conceitual da vulnerabilidade e da SM em um município catarinense.

\section{Metodologia}

Investigação com abordagem qualitativa e descritiva (Silva, \& Fossá, 2015). Realizada em um município catarinense, com colonização alemã, italiana e polonesa, possui pouco mais de 11.000 habitantes. A atividade econômica predominante é agrícola, prevalecendo pequenas propriedades de subsistência e comercialização de excedentes (Instituto Brasileiro de Geografia e Estatistica [IBGE], 2017).

As taxas de morte por suicídio em SC apresentam variações significativas dentre as distintas regiões, os municípios com maior taxa de mortalidade têm a similaridade de serem tipicamente rurais com média populacional 8.000 habitantes. SC é dividida em 16 macrorregiões de saúde, a do município estudado apresentou a média da taxa de mortalidade por suicídio de 10,5, entre 2012 e 2017 (DIVE, 2019).

APS do município tem quatro Unidades Básicas de Saúde (UBS), cinco equipes de Estratégia de Saúde da Família (ESF), uma Equipe de Saúde Mental (ESM) e uma equipe de Núcleo de Apoio à Saúde da Família (NASF), juntas cobrem $100 \%$ da população.

Os participantes foram todos os profissionais de nível superior da secretaria de saúde atuantes na APS do município, naquele momento 19 profissionais, os quais foram selecionados por meio de busca ativa, seguindo os critérios de inclusão: estar alocado em um dos locais do estudo por no mínimo seis meses; prestar assistência direta/indireta a saúde das pessoas com CS.

A coleta de dados ocorreu entre agosto e setembro de 2018, mediante entrevista semiestruturada individual, com prévio agendamento, em local reservado e seguindo roteiro previamente examinado por especialistas, o qual apresentava questões abertas que permitiram a compreensão sobre a experiência e o conhecimento dos profissionais no cuidado a pessoas com comportamento suicida, como esses profissionais identificam tal comportamento, como percebem o preparo para prestar esse cuidado, as dificuldades, redes de apoio, ações e serviços disponíveis.

As entrevistas foram gravadas, transcritas na integra e submetidas à validação de conteúdo pelos participantes. Os dados foram organizados com auxílio do software ATLAS.ti 7.5.6 (QualitativeResearchandSolutions); analisados com base na Análise de Conteúdo, a qual prevê como critério metodológico pré-análise, exploração do material, tratamento dos resultados e interpretação (Silva, \& Fossá, 2015).

Obedecidas as diretrizes/normas da Resolução n 466/2012 (Brasil, 2012). Pesquisa aprovada por Comitê de Ética em Pesquisa, sob parecer $n^{\circ} 2.733 .880$ de 25/06/2018. O anonimato dos participantes foi assegurado, sendo mencionados com a letra S (sujeito), seguido de numeral distribuído aleatoriamente. 


\section{Resultados}

Participaram do estudo os 19 participantes elegíveis, sendo: uma assistente social; um administrador; uma dentista; cinco enfermeiras; duas farmacêuticas, seis médicos; uma nutricionista; e dois psicólogos. Dentre estes, 11 atuam na ESF, oito no NASF, cinco na ESM e dois na gestão da APS municipal. Ainda, quatro destes ocupam cargos em outras secretarias do município. Sinaliza-se que 11 dos 19 profissionais da ESF, NASF e ESM compunham mais de uma equipe.

Emergiram três categorias da organização/análise dos dados, a primeira diz respeito à "Percepção do profissional da APS sobre o CS", seguida da "Abordagem e cuidado à pessoa com CS na APS" e por fim "Aspectos socioculturais e de infraestrutura para o atendimento às pessoas com CS".

\section{Percepção do profissional da APS sobre o CS}

Constata-se que os profissionais entendem o CS como um sofrimento severo, na maioria precedido/acompanhado por transtornos mentais, sintomas depressivos que podem se manifestar como tristeza, agressividade, rompimentos de vínculo, isolamento social, abuso de medicação e pensamentos de morte.

Em visita domiciliar a uma paciente com tentativas prévias de suicídio, ouvi relatos de sofrimento severo, falta de sentido para vida. Identificamos laços rompidos, problemas no trabalho, vínculo familiar quase inexistente. Ela se recolheu e deixou de existir! (S9)

Pode apresentar agressividade, isolamento social. (S14)

Uma situação em particular frustrou porque não detectei, às vezes é muito sutil. Com os anos alguns atendimentos nos marcam/ensinam! (S13)

Quando questionados acerca da etiologia do CS, a totalidade dos participantes apontou múltiplas e particulares causas. Reforçaram a ligação com transtornos mentais, uso de substâncias e outras motivações.

São muitas situações, cada um com complicações particulares. Estresse, relacionamento familiar/social conflituoso, bullying que conduz a pensamentos de suicídio de crianças/adolescentes. Doenças psiquiátricas e abuso de substâncias/medicamentos quase sempre presentes. (S1)

A angústia, o sofrimento imensurável sem conseguir formular compreensão. Pode se configurar como algo traumático, um evento/perda, que mude radicalmente sua vida. Um desamparo severo! Na tentativa da homeostase, a opção é deixar de existir. (S9)

Os participantes concordam que a identificação precoce aumenta as chances de êxito no cuidado e adesão ao tratamento, mas compõe o primeiro obstáculo a ser transposto, pois está envolto por complexidades e demanda profissionais com sentidos aguçados e sensibilizados para ler os sinais e as distintas formas, nem sempre, explicitamente, declaradas pelas pessoas com CS.

Eles se isolam, param de vir às consultas, param com medicamento, muitas vezes os familiares que identificam e 
trazem as queixas, quando o paciente vem para a consulta conversamos e entendemos o que está acontecendo. $O$ relato vem quando questionamos, espontaneamente é raro! (S8)

Identificamos pelas marcas no corpo ou frases de alerta: Minha vida não tem sentido. Quero dormir e não acordar. Quando verbaliza, cabe ao profissional direcionar os questionamentos e entender o que aquela fala representa. Toda atenção é necessária. (S4)

\section{Abordagem e cuidado à pessoa com CS na APS}

Identificou-se que a abordagem profissional é direcionada pelas experiências prévias. Quando se trata de novas situações, os primeiros esforços são em fazer o paciente falar, conversar, escutar e entender as motivações com o objetivo de criar vínculo e identificar necessidades.

Uma vez, chamei atenção de uma mãe, estava atendendo seu filho e de repente ela começou a chorar, contou que havia tentado suicídio. Hoje tenho mais cuidado ao abordar, me sensibilizar, não sabemos o que elas passam fora daqui. (S6)

A médica chamou a enfermeira, ficamos com ele um longo tempo, conversamos/tentamos entender, fazer vínculo, explicamos os caminhos que poderíamos ofertar, dentre eles o CAPS, ligamos e conseguimos atendimento no mesmo dia. As consultas na APS seguiram em paralelo às do CAPS. (S11)

De forma geral, cada profissional tem um membro da equipe como referência para colaborar no manejo, bem como uma rede de apoio municipal. Independente de situações novas ou reincidentes, tratadas ou não, quando identificada situação de risco optam sempre que possível, pelo atendimento conjunto, especialmente entre médico, enfermeiro e psicólogo.

Minha referência é a enfermeira, tem muita experiência em saúde mental, conhece a população. (S6)

O apoio é meu médico, ou ligo para as outras equipes e colegas da atenção básica. (S10)

Nas situações que perpassam a APS, há encaminhamentos ao CAPS, SAMU, unidade de pronto atendimento do município e referência para internação psiquiátrica em hospital geral. Foi valorizado o envolvimento da família/amigos na aceitação, adesão e continuidade do cuidado, muitos pacientes abandonam o tratamento.

Quando existe plano de suicídio o atendimento é feito pelo psicólogo ou encaminhamos ao CAPS, na mediação direta com uma tentativa de suicídio chamamos SAMU. (S15)

A família procura internação, não sabe como lidar, tento conscientizar que após a internação ela volta para a família e precisa de cuidados. Esse primeiro choque, eles entendem que precisam participar, auxiliar na medicação, incentivar, levar as consultas. (S19)

Identificou-se a dificuldade em concretizar alguns encaminhamentos aos demais níveis da RAS constituindo entraves para o adequado manejo, principalmente no que tange as dificuldades e até negativas para alguns encaminhamentos em se 
tratando de situações agudas.

Por vezes manejamos na ESF, com o auxílio do NASF. Cada caso tem suas particularidades, alguns são mais complicados, especialmente quando a família não está engajada no tratamento. Não podemos contar com nossa referência em internação psiquiátrica, não aceitam os encaminhamentos. (S7)

Conversamos com os médicos e a psicóloga. Ela fica pouco tempo aqui, mas temos! Tem o NASF, ligamos para o CAPS e conversar sobre os casos que acompanham. Se precisamos de internação conversamos com a gestão, não é sempre que conseguimos devido à falta de estrutura a nivel estadual/nacional para os casos mais graves; ou conseguimos internação e logo voltam sem um tratamento terapêutico, é uma rotatividade grande de leitos. (S8)

Quando questionados sobre o preparo/segurança para atender as pessoas com CS, os participantes expressaram receio de "deixar algo passar" ou não adotarem a melhor conduta. Eles reconhecem a corresponsabilização do profissional/família/sistema de saúde em cada tratamento ou situação de risco, ainda que, também, tenham sinalizado uma dicotomia e consequente fragmentação desta corresponsabilização.

Pergunto: se negar ajuda quem vão procurar? APS de um município pequeno tem obrigação de fazer isso sozinha? Penso que não! Mas, se não fizermos, quem vai auxiliar essas famílias? Muitos dão as costas, nós tentamos ajudar. (S19)

A oferta de serviços na APS municipal a esse público é constituída, basicamente, por atendimentos clínicos com médicos/enfermeiros/psicólogos, e por vezes não ocorrem na frequência/tempo adequados. Mesmo assim, é o único município da região geopolítica com ESM.

Contamos com consultas do médico, enfermeira e psicóloga. Temos grupo de caminhada, yoga e auriculoterapia para a comunidade. Tentamos vincular o paciente nessas atividades, não temos grupos específicos. (S7)

Um participante sinalizou a vigilância epidemiológica como parte destes atendimentos; os demais não verbalizaram questões acerca da importância das notificações dos casos de tentativas de suicídio ou suicídios.

\section{Aspectos socioculturais e de infraestrutura para o atendimento às pessoas com CS}

Analisou-se, inicialmente, a questão do predomínio da colonização alemã e italiana, formada majoritariamente por famílias de pequenos agricultores que permanecem em suas propriedades, permeados por estigmas e pré-conceitos.

É uma cidade pequena com alto índice de suicídios há anos. A pressão social é grande, a rotina nas pequenas propriedades agrícolas é pesada. Não têm tempo para procurar ajuda, o estereótipo da pessoa ideal e bem sucedida é rígido; questões sociais/culturais favorecem o aparecimento do CS. (S18)

A pressão social quanto aos padrões e estereótipos, modelando a "pessoa perfeita", o sucesso profissional, o modo de agir em sociedade, as características ideais da beleza, podem gerar frustrações e sentimentos de rejeição, fracasso, infelicidade e deslocamento social. 
Temos padrões impostos pelo mercado, a forma do corpo, a estrutura familiar. Não se encaixar leva muitas pessoas à CS. É diferente para cada pessoa, envolve estrutura familiar, escolaridade, emprego, questões envoltas à sexualidade. Vivemos em um município com colonização alemã, culturalmente fechada, machista e preconceituosa.

Ainda, constatou-se que no cuidado ofertado à pessoa com CS pela APS do município, a infraestrutura de atendimento possui como fortalezas: a presença de uma ESM; a possibilidade de realizar atendimentos compartilhados e multiprofissionais; a rede de apoio estabelecida entre os profissionais das distintas equipes atuando sensibilizados, colaborativos e enfrentando às dificuldades.

Desenvolvi depois de formada, com ajuda dos colegas, diferentes formas de questionar/entender o que a pessoa sente e sua representação. Tento fazer vínculo, deixá-la à vontade para contar, procuro outro profissional para ajudar...

Evoluímos nos últimos anos, somos o único município da região com ESM. Se tivéssemos condições de fazer visitas, matriciamentos como no início, e um CAPS mais aberto, seria ideal. (S17)

Em contrapartida, dentre as fragilidades que impactam na adesão ao tratamento, foram referidas: ausência de busca ativa por parte da APS; oferta insuficiente de transporte para pacientes das áreas rurais, que não tem meios próprios de se locomoverem, seja para as consultas na APS, ou CAPS que se localiza fora do município; falta de matriciamento; e ausência de inovações no serviço como grupos terapêuticos e ações de prevenção e conscientização.

Deveria ser prioridade melhorar o acesso. Esse paciente precisa de acompanhamento psicológico, de busca ativa se faltar. Paciente CAPS tem que ser levado para depois ir sozinho. (S2)

A ESM atendia a demanda emergencial, podiam não estar no local, mas direcionavam e na primeira oportunidade acolhiam. Tínhamos busca ativa dos faltosos. Há alguns meses a ESM está enfraquecida, não realiza visitas domiciliares/matriciamento! (S3)

Os profissionais apontaram a sobrecarga de trabalho gerada pelo acúmulo de funções entre ESF, NASF e ESM como situação que corrobora com as deficiências expostas.

\section{Discussão}

O primeiro passo no cuidado ao CS é identificar as pessoas em risco. Contudo, na maioria dos casos, a identificação do CS se dá mediante relato verbal da pessoa ou alguém próximo; em diversas situações os sujeitos não conseguem perceber os sinais, refletindo no sentimento de frustração e autocrítica com relação ao despreparo profissional. Nesta direção, estudo que abordou CS em idosos, sinalizou que este se expressa por comunicações verbais, mas também por um conjunto de sinais/comportamentos passíveis de serem detectados. O profissional deve estar sensibilizado para reconhecer e interagir com a pessoa em sofrimento (Minayo, Figueiredo, \& Mangas, 2019).

Os participantes reconhecem a multicausalidade e manifestações do CS. Ainda assim, apontaram dificuldades para identificar quando não verbalizado. Logo, a ideia foi explorar a habilidade profissional para identificação e interpretação de 
situações-problema e vulnerabilidades por meio da SM. Estudos sugerem que a SM seja promovida através de programas educacionais que enfatizem o "como lidar" com problemas éticos que podem resultar em sofrimento, por acreditar que constituem uma forma de aperfeiçoamento (Nora, Schaefer, Álvarez, \& Ramos, 2016; Nora, Zaboli, \& Vieira, 2017).

Mas, a sensibilidade não é só uma questão de perceber. É componente pessoal adquirido pela experiência cotidiana e em constante aprimoramento, que nos auxilia a compreender o significado moral em uma determinada situação e leva-nos a uma tomada de decisão ética, cautelosa e responsável no exercício profissional (Nora, Zaboli, \& Vieira, 2017).

Conforme os profissionais percebem sinais de risco para o CS, são desencadeados esforços para que a pessoa em situação de vulnerabilidade possa expor suas aflições, motivações e necessidades, estabelecendo vínculo e fortalecendo a relação profissional-paciente-rede. O tipo de abordagem, de resposta das instituições envolvidas e da participação da família/sociedade no processo de cuidado podem romper, gerar, perpetuar e transformar vulnerabilidades. Enfim, os participantes afirmaram não saber lidar com um "pedido de rede" negado, que além de perpetuar algumas vulnerabilidades sociais e estruturais, promovem sofrimento no profissional e na pessoa com CS.

A SM está presenta na percepção e tomada de decisões éticas, mas quando não é possível efetivar a decisão tomada pode gerar sofrimento moral nos profissionais, consolidando vulnerabilidades no cuidado à pessoa em sofrimento. Isto é, as fragilidades organizacionais do sistema de saúde e as demandas cotidianas dos serviços constituem uma prática desafiadora, porque se tornam condições opostas aos preceitos éticos e comprometem a eficácia no manejo no cuidado adequado (Cardoso et al., 2016). Profissionais que lidam com tais dificuldades sofrem as consequências deste processo de deliberação e vivenciam conflitos relacionados com os resultados da sua atuação (Nora, Zaboli, \& Vieira, 2017; Ramos et al., 2016).

O processo de sofrimento moral ocorre pela percepção da existência de um problema que demandaria um adequado direcionamento, e isto, por algum motivo, seria obstruído. Logo, perceber o problema está atrelado ao repensar a prática (Ramos et al., 2016). Nesta perspectiva, sempre que possível, os participantes optaram pelo atendimento compartilhado com outro profissional da rede municipal acreditando que, a soma dos distintos saberes, sustenta uma tomada de decisões mais adequada.

A capacidade para a detecção precoce e prevenção do suicídio, pede o enfrentamento dos estigmas/tabus relacionados ao assunto (Marcolan, \& Silva, 2019). O cenário deste estudo mostrou-se desafiador, constituído por tabus/estigmas relacionados ao aparecimento ou agudização de CS. Esta afirmação considera os aspectos sócios culturais predominantes no município, isto é, os moradores da região são remanescentes de uma cultura onde honrar os compromissos e alcançar o sucesso econômico são valores básicos, passados de geração em geração, também, priorizados pelas exigências do capitalismo (Meneghel, \& Moura, 2018).

O fato de não honrar compromissos, perda de terras, desemprego ou fracasso financeiro podem constituir, para estes pequenos agricultores, determinantes para o suicídio, assim como gatilhos para o ato. A angústia pode levar a pessoa a perder a esperança, cujo desespero tem na morte uma possível saída (Meneghel, \& Moura, 2018; Cabral, \& Oliveira, 2018).

A agricultura familiar implica na dedicação quase que exclusiva às atividades laborais, é comum o trabalho infantil, de mulheres e idosos. Ausência do lazer pode desencadear depressão, apatia, conflitos e condutas autodestrutivas. Ainda, a indiferença frente ao sofrimento psíquico e a tendência em desqualificá-lo, considerando-o como "frescura" para fugir do trabalho. O CS é tido como um sinal de fraqueza/inferioridade (Meneghel, \& Moura, 2018; Cabral, \& Oliveira, 2018).

Nesse âmbito, abordagem educacional responsável ostenta posição insubstituível como fator de prevenção/proteção ao suicídio. A população vulnerável não deve ficar à mercê de seus próprios esforços, mas sim, ser priorizada em intervenções preventivas que incluam famílias/amigos/comunidade. A conscientização e estimulação de ações comunitárias preventivas são necessárias para a mudança da realidade atual (Lucas, Costa, \& Souza, 2017; Minayo, Figueiredo, \& Mangas, 2019). 
Na contramão, temos a insuficiente infraestrutura e ofertas de ações em saúde na APS municipal. Somando barreiras culturais, dificuldade de acesso aos serviços, sobrecarga de trabalho vivenciada pelos profissionais e carência de inovação às adversidades atreladas ao tratamento proposto, totalizam obstáculos sociais/organizacionais de complicada resolução. A falta de continuidade do cuidado, articulada a problemas relacionados aos encaminhamentos e a organização de fluxos reforça a vulnerabilidade do usuário e do próprio profissional (Barth et al., 2019).

O exercício do profissional em saúde no contexto do cuidado ao CS da APS, apresentou-se permeado por problemas éticos reconhecidos por meio da SM. Estes caracterizaram-se por uma sutileza e pela naturalização da violência nas relações interpessoais, apresentando alta frequência e baixa intensidade, que dificultam sua percepção pelos profissionais (Nora, Zaboli, \& Vieira, 2017).

Embora os profissionais objetivem o bem do paciente, estudos (Lima, \& Gondim, 2019; Ferracioli et al., 2019; Minayo, Fiqueiredo, \& Mangas, 2019); Marcolan, \& Silva, 2019; Meneghel, \& Moura, 2018; Cabral, \& Oliveira, 2018; Albuquerque, Boeira, Lima, \& Ayres, 2019) indicam relatos de práticas que degradam e desumanizam os usuários, e que se agravam quando trata-se da pessoa com CS. Esta situação interfere, negativamente, nos cuidados em saúde, aumenta o sofrimento e o risco de nova tentativa de suicídio, e conduz à percepção de que há um entendimento silencioso, uma autorização implícita para a perpetração desses atos nas instituições (Albuquerque, Boeira, Lima, \& Ayres, 2019).

A subnotificação dos casos de suicídio e tentativa de suicídio, consubstancia-se em uma falha ética/profissional/institucional, impacta na carência de recursos, programas e ações de prevenção e na perpetuação das vulnerabilidades envoltas ao CS. Existem irregularidades que fragilizam o sistema de saúde brasileiro, e invisibiliza a realidade, uma vez que os dados epidemiológicos não serão gerados para refletirem em políticas públicas (Melo et al., 2018).

A principal justificativa mencionada para a não notificação é a falta de tempo, produto da sobrecarga de trabalho. A notificação imediata da tentativa de suicídio objetiva gerar dados epidemiológicos, desencadear ações que assegurem o acolhimento e a prestação de cuidados necessários, e oportunizar a adoção de medidas terapêuticas adequadas com o propósito de evitar sua repetição, sendo considerada uma responsabilidade legal dos profissionais de saúde (Melo et al., 2018; Rohling, Ciesca, \& Liebl, 2018).

Este estudo apresenta como limitação a área de abrangência. Mesmo que, tenha sido escolhida uma região cuja demanda de situações vivenciadas com CS seja superior a outros locais no estado, esta escolha pode se constituir num viés, na medida em que outros contextos precisariam ser explorados, para melhor contextualização desta problemática.

\section{Conclusão}

O aporte teórico da SM proporcionou discorrer acerca da complexidade do papel do profissional da APS, e a necessidade do aprimoramento contínuo de habilidades na percepção dos aspectos contextuais que impactam em estratégias de intervenção, em novas práticas e na identificação das barreiras/limites no cuidado ao CS. No entanto, o exercício da SM pode desencadear angústia/sofrimento moral no sujeito trabalhador ao deparar-se com problemas éticos ou barreiras que perpassam sua governabilidade.

Os participantes reconhecem as múltiplas vulnerabilidades e os estigmas/tabus envoltos à pessoa com CS, bem como aspectos relacionados a fatores de risco, etiologia e manifestações. A identificação da pessoa em sofrimento requer atenção e sensibilidade do profissional, para desencadeamento do start imediato para o cuidado.

Durante o cuidado preventivo e manejo terapêutico, foram evidenciadas: dificuldades em sensibilizar familiares/comunidade quanto à relevância da atuação/participação no processo de cura e amenização da dor; inexistência de ações de conscientização no enfrentamento das barreiras culturais; ausência de busca ativa; dificuldade de acesso aos serviços; 
sobrecarga de trabalho dos profissionais e acumulo de funções; e limitações no aprimoramento de estratégias para lidar com as adversidades que emergem no tratamento.

\section{Referências}

Albuquerque, A., Boeira, L., Lima, L., \& Ayres, T. (2019). Os direitos humanos de pacientes em risco de suicídio no Brasil. Cad. Ibero-Am. Direito Sanit, 8(1), 26-35. https://doi.org/10.17566/ciads.v8i1.523

Barth, P. O., Ramos, F. R. S., Barlem, E. L. D., Rennó, H. M. S., Brehmer, L. C. F., \& Rocha, J. M. (2019). Generating situations of moral distress in primary care nurses. Rev. Bras. Enferm, 72(1), 35-42. https://doi.org/10.1590/0034-7167-2018-0378

Brasil. Ministério da Saúde (BR). Diretrizes e normas regulamentadoras de pesquisa envolvendo seres humanos. Resolução nº 466,12 de dezembro de 2012. Brasília; 2012.

Cabral, M. G., \& Oliveira, I. R. B. (2018). A dissimulação é um dever quando a sinceridade é um perigo: o suicídio como busca do alívio. Rev Cientefico, 18(37), 99-119. https://cientefico.emnuvens.com.br/cientefico/article/view/328/353

Cardoso, C. M. L., Pereira, M.O., Moreira, D.A., Tibães, H. B. B., Ramos, F. R. S., \& Brito, M. J. M. (2016). Moral distress in family health strategy: experiences expressed by daily life. REEUSP, 50, 89-95. http://dx.doi.org/10.1590/S0080-623420160000300013

Dive, Diretoria de Vigilância Epidemiológica. Secretaria de Estado da Saúde de Santa Catarina. (2019). Suicídio. Perfil epidemiológico das tentativas e óbitos por suicídio em Santa Catarina e a Rede de Atenção à Saúde (2012-2017). http://www.dive.sc.gov.br/barrigaverde/pdf/BarrigaVerde\%20Suicidio.pdf.

Ferracioli, N. G. M., Oliveira-Cardoso, E. A., Vedana, K. G. G., Pillon, S. C., Miasso, A. I., Souza, J., Risk, E. N., \& Santos, M. A. (2019). Os bastidores psíquicos do suicídio: uma compreensão psicanalítica. Rev NESME, 16(1), 1-17. 10.32467/issn.1982-1492v16n1p17-28

Ibge, Instituto Brasileiro de Geografia e Estatistica. (2017). IBGE Cidades. https://cidades.ibge.gov.br/

Lima, F. N., \& Gondim, D. S. M. (2019). Hereditariedade e repetição no ato suicida: uma abordagem psicanalítica. ISJ, 6(5),354-69. http://dx.doi.org/10.17115/2358-8411/v6n5a24

Luca, L. A. F., Costa, D. A. O., \& Souza, R. M. (2017). Ideação suicida em adolescentes de 15 a 18 anos estudantes do ensino médio da mcrorregião de São Carlos/SP. Brazilian Journal of Forensic Sciences, Medical Law and Bioethics, 6(4), 475-484. http://dx.doi.org/10.17063/bjfs6(4)y2017475

Machado, I. L. O., \& Albuquerque, A. (2019). Papel do Estado quanto à vulnerabilidade e proteção de adultos com deficiência intelectual. Cad. Ibero Am. Direito Sanit, 8(1), 65-79. http://dx.doi.org/10.17566/ciads.v8i1.505

Marcolan, J. F., \& Silva, D.A. (2019). O comportamento suicida na realidade brasileira: aspectos epidemiológicos e da política de prevenção. Revista M., 4(7), 31-44. http://dx.doi.org/10.9789/2525-3050.2019.v4i7.31-44

Melo, M. A. S., Coleta, M. F. D., Coleta, J. A. D., Bezerra, J. C. B., Castro, A. M., Melo, A. L. S., Teixeira, R. A. G., Gomes, D. B., \& Cardoso, H. A. (2018). Percepção dos profissionais de saúde sobre os fatores associados à subnotificação no Sistema Nacional de Agravos de Notificação(Sinan). Rev. Adm. Saúde, 18(71), 1-17. http://dx.doi.org/10.23973/ras.71.104

Meneghel, S. N., \& Moura, R. (2018). Suicídio, cultura e trabalho em município de colonização alemã no sul do Brasil. Interface, 5(22), 1135-1146. https://doi.org/10.1590/1807-57622017.0269

Minayo, M. C. S., Figueiredo, A. E. B., \& Mangas, R. M. N. (2019). Estudo das publicações científicas (2002-2017) sobre ideação suicida, tentativas de suicídio e autonegligência de idosos internados em Instituições de Longa Permanência. Ciênc. \& Saúde Coletiva, 24(4), 1393-1404. https://doi.org/10.1590/1413-81232018244.01422019

Naghavi, M. (2019). Global, regional, and national burden of suicide mortality 1990 to 2016: systematic analysis for the Global Burden of Disease Study 2016.BMJ, 364(194), 01-11. https://doi.org/10.1136/bmj.194

Nora, C. R. D., Zoboli, E. L. C. P., \& Vieira, M. M. (2017). Moral sensitivity in primary health care nurses. Rev Bras Enferm, 70(2), 308-316. https://doi.org/10.1590/0034-7167-2016-0453

Nora, C. R. D., Schaefer, R., Álvarez, E. J., Ramos, M. D. (2016). Sensibilidade Moral e fatores relacionados: percepção de enfermeiros. Cogitare Enferm, 21(4), 01-08. http://dx.doi.org/10.5380/ce.v21i4.47410

Oliveira, L. R., \& Benedetti, A. O. C. (2018). Suicídio em Mato Grosso - Brasil: 1996 a 2015. J. Health Biol Sci, 6(4), 391-398. http://dx.doi.org/10.12662/2317-3076jhbs.v6i4.1763.p391-398.2018

Prata, N. I. S. S., Groisman, D., Martins, D. A., Rabello, E. T., Mota, F. S, Jorge, M. A., Nogueira, M. L., Calicchio, R. R., \& Vasconcelos, R. V. (2017). Saúde mental e atenção básica: território, violência e o desafio das abordagens psicossociais. Trab. Educ. Saúde, 15(1), 33-53. http://dx.doi.org/10.1590/19817746-sol00046

Ramos, F. R. S., Barlem, E. L. D., Brito, M. J. M., Vargas, M. A., Schneider, D. G., \& Brehmer, L. C. F. (2016). Referencial conceitual para o estudo da aflição moral em enfermeiros. Texto contexto-enferm, 25(2), e4460015. https://doi.org/10.1590/0104-07072016004460015

Ribeiro, N. M., Castro, S. S., Scatena, L. M., \& Haas, V. J. (2018). Análise da tendência temporal do suicídio e de sistemas de informações em saúde em relação às tentativas de suicídio. Texto Contexto Enferm, 27(2), e2110016. https://doi.org/10.1590/0104-070720180002110016 
Research, Society and Development, v. 10, n. 4, e43810414282, 2021

(CC BY 4.0) | ISSN 2525-3409 | DOI: http://dx.doi.org/10.33448/rsd-v10i4.14282

Rohling, B. S. V., Ciesca, D., \& Liebl, G. (2018) Projeto vida: integração da vigilância epidemiológica e setor da saúde mental frente às tentativas de suicídio em Fraiburgo, Santa Catarina, 2014-2017. Epidemiol. Serv. Saúde, 27(3), 1-8. https://doi.org/10.5123/s1679-49742018000300014

Sartorino, B. D., Campos, C. F., Chicata, L. C. O., Campos, M. A., Matos, M. S. C., Nunes, R. M. C. M., \& Vidal, C. E. L. (2018). Atitudes de profissionais da saúde em relação ao comportamento suicida. Cad. Saúde colet., 26(4), 369-377. https://doi.org/10.1590/1414-462x201800040191

Silva, B. F. A., Prates, A. A. P., Cardoso, A. A., \& Rosas, N. (2018). O suicídio no Brasil contemporâneo. Soc. Estado, 33(2), 565-579. https://doi.org/10.1590/s0102-699220183302014

Silva, A. H., \& Fossá, M. I. T. (2015). Análise de conteúdo: exemplos de aplicação da técnica para análise de dados qualitativos. Qualitas Rev Eletr, 17(1), 114. http://dx.doi.org/10.18391/qualitas.v16i1.2113 